\title{
THEOREMS OF KREIN-MILMAN TYPE FOR CERTAIN CONVEX SETS OF OPERATORS
}

\author{
BY \\ P. D. MORRIS AND R. R. PHELPS( ${ }^{(1)}$
}

\begin{abstract}
Let $M$ be a real (or complex) Banach space and $C(Y)$ the space of continuous real (or complex) functions on the compact Hausdorff space $Y$. The unit ball of the space of bounded operators from $M$ into $C(Y)$ is shown to be the weak operator (or equivalently, strong operator) closed convex hull of its extreme points, provided $Y$ is totally disconnected, or provided $M^{*}$ is strictly convex. These assertions are corollaries to more general theorems, most of which have valid converses. In the case $M=C(X)$, similar results are obtained for the positive normalized operators. Analogous results are obtained for the unit ball of the space of compact operators (this time in the operator norm topology) from $M$ into $C(Y)$.
\end{abstract}

Introduction. The reason the Krein-Milman theorem does not immediately yield the above results is, of course, the fact that the closed convex sets we are considering are compact only in very special cases. On the other hand, we are greatly helped by the fact that the range space is $C(Y)$, since the space of bounded linear operators from $M$ may then be represented [4] in a simple way as the space of weak* continuous functions (or norm continuous, in the case of compact operators) from $Y$ into $M^{*}$. The unit ball $S$ of operators may be identified with those weak* continuous maps which carry $Y$ into the unit ball $S\left(M^{*}\right)$ of $M^{*}$. While it is generally a nontrivial task to characterize the functions which correspond to extreme points of $S$, it is easily verified that those maps which carry $Y$ into the set ext $S\left(M^{*}\right)$ of extreme points of $S\left(M^{*}\right)$ define extreme operators and we refer to the latter as nice operators. In some special cases, every extreme operator is nice. When this is not the case, however, we concentrate on the nice operators, so that our problem becomes one of showing that there exist "sufficiently many" continuous functions from $Y$ into $Z=\operatorname{ext} S\left(M^{*}\right)$. It is clear that this requires the placing of topological restrictions on $Y$ or $Z$, and in $\$ 1$ we consider briefly the needed properties.

In $\$ 2$ it is shown that $S$ is the weak operator-closed. convex hull of its extreme points when any one of the following holds: (i) $Y$ is totally disconnected and $M$

Received by the editors October 20, 1969.

AMS Subject Classifications. Primary 4610, 4625, 4725; Secondary 4745.

Key Words and Phrases. Bounded linear operators, convex sets of operators, extreme operators, Banach space-valued functions, linear operators into $C(X)$, compact linear operators.

(1) Work on this paper was supported in part by NSF Grant GP 8759 for the first named author, and by NSF Grant GP 11469 for the second.

Copyright (C) 1970, American Mathematical Society 
is arbitrary; (ii) $M^{*}$ is strictly convex and $Y$ is arbitrary; (iii) the scalars are complex, $Y$ is arbitrary and $M$ is an $L$-space; (iv) the scalars are complex, $Y$ is arbitrary, and $M=C(X)$, with $X$ arcwise connected. In connection with (i), it is proved in $\S 3$ that total disconnectedness is also a necessary condition in case $M=C(X)$, $X$ compact metric, and the scalars are real.

In the latter part of $\S 2$, we take $M=C(X)$, and we replace $S$ by one of the sets:

$$
K_{0}=\{T: T \geqq 0, T 1 \leqq 1\} \quad \text { or } \quad K_{1}=\{T: T \geqq 0, T 1=1\} .
$$

It is proved, for example, that $K_{0}$ is the weak operator-closed convex hull of its extreme points if and only if $Y$ is totally disconnected.

In $\S 3$, we prove partial converses to some of the results of $\S 2$.

In $\S 4$, we take $S$ to be the unit ball of the compact operators from $M$ into $C(Y)$, in the operator norm topology. For general spaces $M$, our main result (Theorem 4.4 ) is that $S$ is the operator norm-closed convex hull of its extreme points if the unit ball of $M^{*}$ is the norm-closed convex hull of its extreme points and if either:

(1) the set of extreme points of $M^{*}$ is arcwise connected (in particular, if $M^{*}$ is strictly convex); or

(2) $Y$ is totally disconnected.

In fact, what is actually proved is that $S$ is the operator norm-closed convex hull of the nice compact operators. In Corollary 4.8, we obtain some partial converses.

In case $M=C(X)$, our results are quite complete. We prove (Theorem 4.5) that all the extreme compact operators are nice and then prove the following: $S$ is the operator norm-closed convex hull of its extreme points if and only if $Y$ is totally disconnected and $X$ contains no nonvoid perfect subsets.

In Corollary 4.2 we give conditions under which the unit ball of $C(Y, N)$ ( $Y$ compact Hausdorff, $N$ a Banach space) is the norm-closed convex hull of its extreme points. This result includes a number of previously known theorems on this question.

In $\S 5$ we investigate the set $\mathscr{E}$ of positive extension operators of norm 1 from $C(X)$ to $C(Y)$, where $X$ is a closed subset of a compact metric space $Y$. Our result is that $\mathscr{E}$ is the weak operator-closed convex hull of its extreme points if and only $Y \backslash X$ is totally disconnected.

We now establish some terminology and notation. First, the scalar field may be either the real or complex numbers unless explicitly restricted to be one or the other. For $X$ a compact Hausdorff space and $M$ a Banach space, $C(X, M)$ denotes the space of all norm-continuous mappings from $X$ into $M$, normed by:

$$
\|f\|=\sup \{\|f(x)\|: x \in X\} .
$$

In case $M$ is the scalar field $\boldsymbol{R}$ or $C$, we write simply $C(X)$ unless it is material which field we are working over.

For $M$ a Banach space, $M^{*}$ denotes the space of bounded linear functionals 
on $M$, normed in the usual way. If $x \in M$ and $x^{*} \in M^{*},\left\langle x^{*}, x\right\rangle$ denotes the value of $x^{*}$ at $x$. The space $C(X)^{*}, X$ compact Hausdorff, is identified with the space of finite regular Borel measures on $X$ and, for $\mu \in C(X)^{*}, f \in C(X)$, we will sometimes write $\int f d \mu$ instead of $\langle\mu, f\rangle$. If $N$ and $M$ are Banach spaces, then $L(M, N)$, or simply $L$ if no confusion is likely, denotes the space of all bounded linear operators from $M$ into $N$. We consider two topologies on $L$; that given by the uniform norm: $\|T\|=\sup \{\|T x\|: x$ in $M,\|x\| \leqq 1\}$, and the weak-operator topology, as defined e.g. in $[4$, p. 476]. We remark that, in our results, the weak-operator topology can be replaced by the strong-operator topology since the closed convex sets are the same for both topologies [4, Corollary VI. 1.5, p. 477].

If $T$ is in $L(M, C(Y))$, there is a map $T^{*}: Y \rightarrow M^{*}$ defined by

$$
\left\langle T^{*} y, x\right\rangle=(T x)(y), \quad x \in M, \quad y \in Y .
$$

(This is, of course, just the restriction of the adjoint map of $T$ to (the natural embedding of) $Y$ in $C(Y)^{*}$.) The map $T^{*}$ is continuous from $Y$ into the weak* topology of $M^{*}$, is norm continuous if and only if $T$ is compact, and any weak* continuous function $T^{*}: Y \rightarrow M^{*}$ defines a unique operator $T$ in $L(M, C(Y))$, by the above formula [4, p. 490]. Now $\|T\| \leqq 1$ if and only if $T^{*}[Y] \subseteq S\left(M^{*}\right)$, the unit ball of $M^{*}$, and $T$ is called nice provided $T^{*}(Y) \subseteq$ ext $S\left(M^{*}\right)$, the set of extreme points of $S\left(M^{*}\right)$. It is easily verified that a nice operator is always extreme, but the converse is false in general [2]. In the case, however, where $M=C(X), X$ compact metric, and the scalars are real, it is known [2] that all extreme operators are nice. It is an open question whether this remains true for arbitrary compact $X$ or for complex scalars.

1. Property (D) and almost arcwise connected spaces. For $Y$ and $Z$ two topological spaces, let us denote by $Z^{Y}$ the set of all functions from $Y$ to $Z$ and by $C(Y, Z)$ the set of all continuous members of $Z^{Y}$. We make the following:

DEFINITION 1.1. The ordered pair $(Y, Z)$ of topological spaces has property (D) if, whenever $n$ is a positive integer, $U_{1}, U_{2}, \ldots, U_{n}$ are nonempty open subsets of $Z$, and $K_{1}, K_{2}, \ldots, K_{n}$ are pairwise disjoint, nonempty, compact subsets of $Y$, there exists $\varphi$ in $C(Y, Z)$ such that $\varphi\left[K_{i}\right] \subseteq U_{i}, i=1,2, \ldots, n$.

Property (D) is a form of density of $C(Y, Z)$ in $Z^{Y}$. Indeed, it is easily verified that (D) implies that $C(Y, Z)$ is dense in $Z^{Y}$ in the topology of pointwise convergence. Furthermore, (D) is implied by density of $C(Y, Z)$ in $Z^{Y}$ in the compactopen topology [7, p. 221].

We are reluctant to introduce yet another class of general topological spaces, but the notion defined below is both a sufficient (Corollary 2.4) and a necessary condition (Corollary 3.3) for the validity of the kind of theorem we are interested in.

Definition 1.2. The topological space $X$ is almost arcwise connected (a.a.c.) if, for every finite collection $\left\{U_{1}, \ldots, U_{n}\right\}$ of nonempty, open subsets of $X$, there is an arc $\Gamma$ (i.e. a continuous image of the unit interval) in $X$ such that $\Gamma \cap U_{i} \neq \varnothing$, $i=1,2, \ldots, n$. 
Note that every a.a.c. space is connected and that every arcwise connected space is a.a.c. On the other hand, it is not difficult (using the $\sin (1 / x)$ curve) to construct examples in the plane of compact connected spaces which are not a.a.c. and a.a.c. spaces which are not arcwise connected. Although we make no use of the fact, it is interesting to note that $X$ is a.a.c. if and only if $C(I, X)$ is pointwise dense in $X^{I}$, where $I=[0,1]$.

The following result guarantees us a fairly rich supply of pairs $(Y, Z)$ having property (D).

Proposition 1.3. Let $Y$ and $Z$ be Hausdorff topological spaces. Then $(Y, Z)$ has (D) if either of the following conditions hold:

(1) $Y$ is locally compact and totally disconnected; or

(2) $Z$ is a.a.c. and $Y$ is normal.

Proof. The proof that (1) implies (D) is easy and is omitted. Now assume (2) and let $U_{1}, U_{2}, \ldots, U_{n}, K_{1}, K_{2}, \ldots, K_{n}$ be as in the definition of property (D). Let $\Gamma=h[I]$ be an arc in $Z$, where $I=[0,1]$ and $h: I \rightarrow Z$ is continuous, such that $\Gamma \cap U_{i} \neq \varnothing, i=1,2, \ldots, n$. For each $i$, choose $t_{i}$ in $I$ such that $h\left(t_{i}\right) \in U_{i}$. Let $f: Y \rightarrow I$ be a continuous map such that $h\left[K_{i}\right]=\left\{t_{i}\right\}, i=1,2, \ldots, n$. Then $\varphi=h \circ f$ is the required map.

It seems very likely that property (D) is actually stronger than pointwise density of $C(Y, Z)$ in $Z^{Y}$, although we have no example. Lemma 3.2 (below) shows that if $(Y, Z)$ is such an example, then $Z$ must be connected but not almost arcwise connected, while $Y$ must not be totally disconnected but must contain no arc. It is not hard to see that (D) is quite a bit weaker than $C(Y, Z)$ being compactopen dense in $Z^{Y}$. For, let $Y=Z=[0,1]$. Then the compact-open topology on $C(Y, Z)$ is the topology of uniform convergence and so $C(Y, Z)$ is complete and thus closed in $Z^{Y}$. That $([0,1],[0,1])$ has $(D)$ is clear from Tietze's theorem, or Proposition 1.3.

2. The bounded operators in the weak-operator topology. Let $M$ be a Banach space and let $Y$ be a compact Hausdorff space. For a nonvoid subset $A$ of $M^{*}$, we denote by $L_{A}$ the set $\left\{T\right.$ in $\left.L(M, C(Y)): T^{*}[Y] \subseteq A\right\}$. The central result of this section is the following:

THEOREM 2.1. Let $M$ and $Y$ be as above. If $Z$ is a bounded nonvoid subset of $M^{*}$ (taken with the weak* topology) such that $(Y, Z)$ has property (D), then $\mathrm{co}^{-} L_{Z}$ $=L_{\mathrm{co}^{-}}{ }_{z}$, where the closure on the left-hand side is weak operator and on the righthand side weak*.

Proof. The inclusion $\mathrm{co}^{-} L_{z} \subseteq L_{\mathrm{co}^{-}}{ }_{z}$ is clear since $L_{\mathrm{co}^{-}}{ }_{z}$ is convex, weak operatorclosed and contains $L_{z}$. To prove the opposite inclusion, let us assume, on the contrary, that there exists $F$ in $L_{\mathrm{co}^{-}}{ }_{z} \mid \mathrm{co}^{-} L_{z}$. Using the Hahn-Banach theorem, we 
see that there exist $m_{1}, m_{2}, \ldots, m_{n}$ in $M, \mu_{1}, \mu_{2}, \ldots, \mu_{n}$ in $C(Y)^{*}$, and $r>0$ such that

$$
\operatorname{Re}\left(\sum_{1}^{n}\left\langle\mu_{j}, F m_{j}\right\rangle\right) \geqq \operatorname{Re}\left(\sum_{1}^{n}\left\langle\mu_{j}, G m_{j}\right\rangle\right)+r
$$

for all $G$ in co- $L_{z}$. For each $j$, we can replace $\mu_{j}$ by $\left(\nu_{j_{1}}-\nu_{j_{2}}\right)+i\left(\nu_{j_{3}}-\nu_{j_{4}}\right)$, where each $\nu_{j_{k}}$ is nonnegative. By then letting $m_{j}$ absorb the coefficient $\pm 1, \pm i$ in each term of $\left\langle v_{j_{1}}, F m_{j}\right\rangle-\left\langle v_{j_{2}}, F m_{j}\right\rangle+i\left\langle\nu_{j_{3}}, F m_{j}\right\rangle-i\left\langle\nu_{j_{4}}, F m_{j}\right\rangle$, we obtain an inequality of the form (1) involving only nonnegative members of $C(Y)^{*}$. We assume that this has already been done so that $\mu_{j} \geqq 0$ for $j=1,2, \ldots, n$. Let $\nu=\sum_{1}^{n} \mu_{j}$. Clearly, each $\mu_{j}$ is absolutely continuous with respect to $\nu$ and, therefore, the Radon-Nikodym derivative $h_{j}$ of $\mu_{j}$ with respect to $\nu$ exists. Certainly, $0 \leqq h_{j} \leqq 1$, a.e. $-\nu$, for all $j$. Now let $\varepsilon$ be a positive number. We shall prove that a contradiction to (1) can be obtained if $\varepsilon$ is taken sufficiently small. For each $j=1,2, \ldots, n$, choose $h_{j}^{\prime}$ in $C(Y)$ such that

$$
\int_{Y}\left|h_{j}-h_{j}^{\prime}\right| d \nu<\varepsilon
$$

We may choose $h_{j}^{\prime}$ so that $0 \leqq h_{j}^{\prime} \leqq 1$. Now let $g=\sum_{1}^{n} h_{j}^{\prime} F m_{j}$ in $C(Y)$. For each $y$ in $Y$, let $\alpha_{y}=F^{*} y \in \mathrm{co}^{-} Z$. Also, let

$$
k_{y}=\sum_{1}^{n} h_{j}^{\prime}(y) m_{j} \in M
$$

Then we have

$$
\left\langle\alpha_{y}, k_{y}\right\rangle=\sum_{1}^{n} h_{j}^{\prime}(y)\left(F m_{j}\right)(y)=g(y) .
$$

Hence, there is an extreme point $\beta$ of the compact convex set $\mathrm{co}^{-} Z$ such that $\operatorname{Re}\left\langle\beta, k_{y}\right\rangle \geqq \operatorname{Re} g(y)$. By Milman's theorem all the extreme points of $\mathrm{co}^{-} Z$ are in the closure of $Z$, so there exists $\gamma$ in $Z$ such that

$$
\operatorname{Re}\left\langle\gamma, k_{y}\right\rangle=\operatorname{Re} \sum_{1}^{n} h_{j}^{\prime}(y)\left\langle\gamma, m_{j}\right\rangle>\operatorname{Re} g(y)-\varepsilon .
$$

Therefore, these exists a relatively weak* open subset $U_{y}$ of $Z$ and an open neighborhood $V_{y}$ of $y$ such that

$$
\operatorname{Re}\left(\sum_{1}^{n} h_{j}^{\prime}(w)\left\langle\lambda, m_{j}\right\rangle\right)>\operatorname{Re} g(w)-2 \varepsilon,
$$

for all $w$ in $V_{y}$ and for all $\lambda$ in $U_{y}$.

The collection $\left\{V_{y}: y \in Y\right\}$ defined in this way forms an open cover of $Y$. Let $\left\{V_{y_{1}}, \ldots, V_{y_{k}}\right\}$ be a finite subcover such that no proper subcollection covers $Y$. By replacing, if necessary, the $V_{y_{i}}$ 's by smaller open sets, we may assume that

$$
\nu\left(\left\{y \in Y: y \text { belongs to more than one } V_{y}\right\}\right)<\varepsilon \text {. }
$$


For each $j=1,2, \ldots, k$, let

$$
H_{j}=V_{y_{j}} \backslash \cup\left\{V_{y_{l}}: l=1,2, \ldots, n ; l \neq j\right\} .
$$

Then each $H_{j}$ is a nonvoid closed set and $H_{j} \cap H_{l}=\varnothing$ if $j \neq l$. Also $\nu\left(Y \backslash \cup H_{j}\right)<\varepsilon$. Since $(Y, Z)$ has property (D), there exists a continuous map $\varphi$ of $Y$ into $Z$ such that $\varphi\left[H_{j}\right] \subseteq U_{y_{j}}$, for each $j$. We have

$$
\operatorname{Re}\left(\sum_{1}^{n} h_{i}^{\prime}(y)\left\langle\varphi(y), m_{j}\right\rangle\right) \geqq \operatorname{Re}(g(y))-2 \varepsilon,
$$

for all $y \in Y$ outside a set $D=Y \backslash \cup H_{j}$ of $\nu$-measure less than $\varepsilon$. Now define $G: M \rightarrow C(Y)$ by: $(G m)(y)=\langle\varphi(y), m\rangle$, for all $m$ in $M$ and for all $y$ in $Y$. Then $G$ is in $L_{z}$ and

$$
\operatorname{Re}\left(\sum_{1}^{n} h_{j}^{\prime}(y)\left(G m_{j}\right)(y)\right) \geqq \operatorname{Re}(g(y))-2 \varepsilon,
$$

for all $y \in Y \backslash D$. Therefore,

$$
\operatorname{Re}\left(\int_{Y \backslash D} \sum_{i}^{n} h_{i}^{\prime} G m_{i} d \nu\right) \geqq \operatorname{Re}\left(\int_{Y \backslash D} g d \nu\right)-2 \varepsilon \nu(Y) .
$$

Hence,

$$
\operatorname{Re}\left(\int_{Y} \sum_{1}^{n} h_{j}^{\prime} G m_{j} d \nu\right) \geqq \operatorname{Re}\left(\int_{Y} \sum_{1}^{n} h_{j}^{\prime} F m_{j} d \nu\right)-2 \varepsilon \nu(Y)-2 n J \varepsilon,
$$

where $J=\max \left\{\left\|m_{i}\right\|: i=1,2, \ldots, n\right\} \cdot \max \{\|\alpha\|: \alpha \in Z\}$. Therefore,

$$
\operatorname{Re}\left(\int_{Y} \sum_{1}^{n} h_{i} G m_{i} d \nu\right) \geqq \operatorname{Re}\left(\int_{Y} \sum_{1}^{n} h_{i} F m_{i} d \nu\right)-2 \varepsilon \nu(Y)-4 n J \varepsilon
$$

Thus

$$
\operatorname{Re}\left(\sum_{1}^{n}\left\langle\mu_{i}, G m_{i}\right\rangle\right) \geqq \operatorname{Re}\left(\sum_{1}^{n}\left\langle\mu_{i}, F m_{i}\right\rangle\right)-2 \varepsilon \nu(Y)-4 n J \varepsilon,
$$

which contradicts (1) if $\varepsilon$ is taken small enough. This completes the proof.

In order to prove the corollaries we are interested in, we specialize the above result as follows:

THEOREM 2.2. Let $M$ be a Banach space and let $Y$ be a compact Hausdorff space. If $\left(Y\right.$, ext $S\left(M^{*}\right)$ ) has property (D) (with ext $S\left(M^{*}\right)$ having the weak* topology), then $S(L(M, C(Y)))$ is the weak operator-closed convex hull of its extreme points.

Proof. Let $Z$ denote ext $S\left(M^{*}\right)$. Then, since $(Y, Z)$ has property (D), $\operatorname{co}^{-} L_{Z}$ $=L_{\mathrm{co}^{-}} \mathrm{z}$, by Theorem 2.1. By a remark made in the introduction, $L_{z} \subseteq \operatorname{ext} S(L)$. Also, $\operatorname{co}^{-} Z=S\left(M^{*}\right)$, by the Krein-Milman theorem. Hence

$$
\mathrm{co}^{-} \operatorname{ext} S(L) \supseteq \mathrm{co}^{-} L_{\mathrm{z}}=L_{\mathrm{co}^{-} \mathrm{z}}=L_{S\left(M^{*}\right)}=S(L),
$$

and the proof is complete. 
CoRollary 2.3. Let $M$ be a Banach space and let $Y$ be a compact Hausdorff space. If $Y$ is totally disconnected or if ext $S\left(M^{*}\right)$ is weak* almost arcwise connected (in particular, if $M^{*}$ is strictly convex), then $S(L(M, C(Y))$ ) is the weak operator-closed convex hull of its extreme points.

Proof. The result follows immediately from Theorem 2.2, Proposition 1.3, and the observation that ext $S\left(M^{*}\right)$ is arcwise connected since $M^{*}$ is strictly convex.

Corollary 2.4. Let $X$ and $Y$ be compact Hausdorff spaces. If $X$ is almost arcwise connected, then $S(L(C(X, C), C(Y, C)))$ is the weak operator-closed convex hull of its extreme points.

Proof. Topologically, ext $S\left(C(X)^{*}\right)$ is $X \times S_{1}$, where $S_{1}$ is the unit circle in the complex plane. Since $X$ is a.a.c., so is $X \times S_{1}$, and the result follows from Corollary 2.3.

The notion of an $L$-space was extended to complex Banach spaces in [18].

CoRollary 2.5. Let $M$ be a complex L-space and let $Y$ be a compact Hausdorff space. Then $S(L(M, C(Y)))$ is the weak operator-closed convex hull of its extreme points.

Proof. It need only be proved that ext $S\left(M^{*}\right)$ is arcwise connected in the weak* topology. We shall actually prove the stronger assertion that ext $S\left(M^{*}\right)$ with the norm topology is arcwise connected. Now $M^{*}=C(W)$, where $W$ is some extremally disconnected compact Hausdorff space (see [19]). Also

$$
\operatorname{ext} S\left(M^{*}\right)=\{f \in C(W):|f(w)|=1 \text { for each } w \in W\}
$$

Let $f \in \operatorname{ext} S\left(M^{*}\right)$ be given. We shall complete the proof by producing an arc from $f$ to $1_{W}$, where $1_{W}$ is the function on $W$ identically equal to 1 . First, decompose $W$ into the union of pairwise disjoint, open-closed sets $\left\{U_{i}: i=1,2, \ldots, n\right\}$ such that $f\left[U_{i}\right]$ is not all of the unit circle $S_{1}$. Define maps $I, u: f\left[U_{1}\right] \rightarrow S_{1}$ as follows: Let $I(z)=z$ and $u(z)=1$, for all $z$ in $f\left[U_{1}\right]$. Then $I$ and $u$ are homotopic maps, that is, there exists a continuous map $H: f\left[U_{1}\right] \times[0,1] \rightarrow S_{1}$ such that $H(\cdot, 0)=I$ and $H(\cdot, 1)=u$. For each $t$ in $[0,1]$, define, for $w$ in $W$,

$$
\begin{aligned}
f_{t}(w) & =f(w), & & w \text { in } \bigcup_{2}^{n} U_{i}, \\
& =H(f(w), t), & & w \text { in } U_{1} .
\end{aligned}
$$

Clearly, each $f_{t}$ is in ext $S\left(M^{*}\right)$, and $t \rightarrow f_{t}$ is an arc in ext $S\left(M^{*}\right)$ from $f$ to $f_{1}$. Repeating this process $n-1$ more times (for $U_{2}, U_{3}, \ldots, U_{n}$ ) and adjoining the resultant arcs gives us the desired arc from $f$ to $1_{W}$.

This completes the proof.

We now turn our attention to sets of positive operators. Let $X$ and $Y$ be compact Hausdorff spaces and let $K_{0}=K_{0}(X, Y), K_{1}=K_{1}(X, Y)$ be the sets of operators from $C(X)$ to $C(Y)$ defined as follows:

$$
K_{0}=\{T: T \geqq 0 \text { and } T 1 \leqq 1\}, \quad K_{1}=\{T: T \geqq 0 \text { and } T 1=1\} .
$$


Both $K_{0}$ and $K_{1}$ are weak operator-closed convex subsets of $L$. Their extreme points have been completely characterized (see [5], [6], and [12]) and these characterizations may be stated as follows:

$$
\text { ext } K_{0}=\left\{T: T^{*}[Y] \subseteq X \cup\{0\}\right\}, \quad \operatorname{ext} K_{1}=\left\{T: T^{*}[Y] \subseteq X\right\},
$$

where we have identified $X$ with its natural embedding in $C(X)^{*}$.

Our results for these two sets are the following:

COROLlary 2.6. Let $X$ and $Y$ be compact Hausdorff spaces. If $Y$ is totally disconnected or $X$ is almost arcwise connected, then $K_{1}(X, Y)$ is the weak operatorclosed convex hull of its extreme points.

Proof. Theorem 2.1 and Proposition 1.3 apply immediately, once we observe that

$$
K_{1}=\left\{T: T^{*}[Y] \subseteq \mathrm{co}^{-} X\right\}=L_{\mathrm{co}^{-} X} .
$$

Corollary 2.7. Let $X$ and $Y$ be compact Hausdorff spaces. Then $K_{0}(X, Y)$ is the weak operator-closed convex hull of its extreme points if, and only if, $Y$ is totally disconnected.

Proof. Suppose $Y$ is totally disconnected. Let $Z=X \cup\{0\}$. Then

$$
\operatorname{co}^{-} Z=\left\{\mu \in C(X)^{*}: \mu \geqq 0 \text { and }\|\mu\| \leqq 1\right\}
$$

and so $K_{0}=L_{\mathrm{co}^{-}} z$. Hence

$$
\mathrm{co}^{-} \text {ext } K_{0}=\mathrm{co}^{-} L_{z}=L_{\mathrm{co}^{-} z}=K_{0},
$$

by Theorem 2.1 and Proposition 1.3. Now suppose $Y$ is not totally disconnected and let $W \subseteq Y$ be a connected set with more than one point. Let $T \in \operatorname{ext} K_{0}$. Then $T^{*}[Y] \subseteq X \cup\{0\}$. Since $T^{*}$ is weak* continuous and 0 is an isolated point of $X \cup\{0\}$, we see that $T^{*}$ either carries all of $W$ to 0 or none of $W$ to 0 . Hence $T 1$ is identically 0 on $W$ or identically 1 on $W$. It follows that $S 1$ is constant on $W$ for every $S \in$ co (ext $K_{0}$ ) and hence for every $S \in \mathrm{co}^{-}\left(\right.$ext $\left.K_{0}\right)$. Therefore, to complete the proof, it suffices to produce $S \in K_{0}$ such that $S 1$ is not constant on $W$. To do this, let $g \in C(Y)$ be such that $0 \leqq g \leqq 1$ and such that $g$ is not constant on $W$. Then fix $x_{0} \in X$ and define $S f=f\left(x_{0}\right) g$, for all $f$ in $C(X)$. It is easily checked that $S$ is in $K_{0}$. Since $S 1=g$ is not constant on $W$, the proof is complete.

3. Partial converses to some of the preceding results. It was pointed out after the definition of property (D) that pointwise density of $C(Y, Z)$ in $Z^{Y}$ is a weaker property than (D). The following is therefore a partial converse to Theorem 2.2. Recall that an operator $T$ was defined in the introduction to be nice if $T^{*}$ maps $Y$ into extreme points.

THEOREM 3.1. Let $M$ be a Banach space and let $Y$ be a compact Hausdorff space. If $S(L(M, C(Y)))$ is the weak operator-closed convex hull of the nice operators, then $C\left(Y\right.$, ext $\left.S\left(M^{*}\right)\right)$ is pointwise dense in (ext $\left.S\left(M^{*}\right)\right)^{Y}$. 
Proof. Let $Z$ denote ext $S\left(M^{*}\right)$. Suppose $U_{1}, \ldots, U_{n}$ are nonvoid weak* open subsets of $Z$ and that $y_{1}, y_{2}, \ldots, y_{n}$ are distinct points of $Y$. We must find a continuous map $\varphi: Y \rightarrow Z$ such that $\varphi\left(y_{i}\right) \in U_{i}, i=1,2, \ldots, n$. We can assume, without loss of generality, that each $U_{i}$ is the intersection with ext $S\left(M^{*}\right)$ of a convex set of the form $S\left(M^{*}\right) \backslash J_{i}$, where $J_{i}$ is a weak* compact convex subset of $S\left(M^{*}\right)$ (see, e.g., the proof of Proposition 6.4 in [14]). Now use the separation theorem to choose $x_{i} \in M$ with $\left\|x_{i}\right\|=1$ and with $\sup \left\{\operatorname{Re}\left\langle x^{*}, x_{i}\right\rangle: x^{*} \in J_{i}\right\}<1$. There is a point $x^{*} \in U_{i}$ such that $\left\langle x_{i}^{*}, x_{i}\right\rangle=1$. Choose $\delta_{i}>0$ so that $\operatorname{Re}\left\langle x^{*}, x_{i}\right\rangle \leqq 1-\delta_{i}$, for all $x^{*}$ in $J_{i}$, and let $\delta=\min \left\{\delta_{i}: i=1,2, \ldots, n\right\}$. Now choose real functions $h_{1}, \ldots, h_{n}$ in $C(Y)$ such that $h_{i} \geqq 0, \sum h_{i} \leqq 1$, and $h_{i}\left(y_{j}\right)=\delta_{i j}$, for all $i$ and all $j$. For $m$ in $M$ and for $y$ in $Y$, define

$$
(\operatorname{Tm})(y)=\sum_{1}^{n} h_{i}(y)\left\langle x_{i}^{*}, m\right\rangle .
$$

Then $T$ is a bounded linear operator from $M$ into $C(Y)$ and, in fact, $T$ is in $S(L(M, C(Y)))$. Therefore, by hypothesis, there exists a nice operator $S$ such that

$$
\operatorname{Re} \sum_{1}^{n}\left(S x_{i}\right)\left(y_{i}\right)>\operatorname{Re} \sum_{1}^{n}\left(T x_{i}\right)\left(y_{i}\right)-\delta / 2
$$

We shall prove that $S^{*}$ is the required map. Now

$$
\left(T x_{i}\right)\left(y_{i}\right)=\sum_{j=1}^{n} h_{j}\left(y_{i}\right)\left\langle x_{j}^{*}, x_{i}\right\rangle=\left\langle x_{i}^{*}, x_{i}\right\rangle=1 .
$$

Hence we have

$$
\operatorname{Re} \sum_{1}^{n}\left\langle S^{*} y_{i}, x_{i}\right\rangle \equiv \operatorname{Re} \sum_{1}^{n}\left(S x_{i}\right)\left(y_{i}\right)>n-\delta / 2 .
$$

Suppose $S^{*}\left(y_{j}\right) \notin U_{j}$, for some $j$. Then $\operatorname{Re}\left\langle S^{*}\left(y_{j}\right), x_{j}\right\rangle \leqq 1-\delta$ and so

$$
n-\delta / 2<\operatorname{Re} \sum_{1}^{n}\left\langle S^{*}\left(y_{i}\right), x_{i}\right\rangle \leqq n-1+1-\delta=n-\delta,
$$

which is absurd. Therefore $S^{*}\left(y_{i}\right)$ is in $U_{i}$ for all $i$, and the proof is complete.

The proof of the following is immediate from the appropriate definitions.

LEMMA 3.2. Let $Y$ and $Z$ be topological spaces such that $C(Y, Z)$ is pointwise dense in $Z^{Y}$.

(1) If $Z$ is not connected, then $Y$ is totally disconnected.

(2) If $Y$ contains an arc, then $Z$ is almost arcwise connected.

The combination of Theorem 3.1 and Lemma 3.2 yields considerable information about the consequences of $S(L(M, C(Y)))$ being the weak operator-closed convex hull of the nice operators. In particular: 
Corollary 3.3. Let $X$ and $Y$ be compact Hausdorff spaces such that $S(L(C(X), C(Y)))$ is the weak operator-closed convex hull of the nice operators.

(1) If the scalars are real, then $Y$ is totally disconnected.

(2) If $X$ is not connected, then $Y$ is totally disconnected.

(3) If $Y$ contains an arc, then the scalars are complex and $X$ is almost arcwise connected.

Proof. (1) Since the scalars are real, ext $S\left(C(X)^{*}\right)$ is topologically a union of two disjoint copies of $X$ and is therefore not connected. An application of Theorem 3.1 and part (1) of Lemma 3.2 completes the proof.

(2) Since $X$ is not connected, neither is ext $S\left(C(X)^{*}\right)$ and the proof is completed as above.

(3) By Theorem 3.1 and part (2) of Lemma 3.2, the space ext $S\left(C(X)^{*}\right)$ is almost arcwise connected and therefore connected. This is only possible when the scalars are complex (see the proof of (1)). Hence, ext $S\left(C(X)^{*}\right)$ is $X \times S_{1}$. Since $X \times S_{1}$ is therefore almost arcwise connected and since continuous images of a.a.c. spaces have the same property, $X$ is almost arcwise connected.

This completes the proof.

Corollary 3.4. Let $X$ and $Y$ be compact Hausdorff spaces with $X$ metrizable. Take the scalar field to be real. Then $S(L(C(X), C(Y)))$ is the weak operator-closed convex hull of its extreme points if and only if $Y$ is totally disconnected.

Proof. Use Corollaries 2.3, and 3.3, and the fact, mentioned in the introduction, that in the present case all extreme operators are nice.

4. Compact operators in the norm topology. For $M$ and $N$ Banach spaces, denote by $K=K(M, N)$ the space of all compact operators from $M$ into $N$. We shall investigate conditions under which $S(K(M, C(Y)))$ is the norm-closed convex hull of its extreme points, where $Y$ is a compact Hausdorff space.

As was noted in the introduction, if $T$ is in $K(M, C(Y))$, then $T^{*}$ is in $C\left(Y, M^{*}\right)$, and it is easily seen that the correspondence $T \leftrightarrow T^{*}$ is a linear isometry between these two spaces. It will be convenient to use this identification at times.

We shall also need to make use of a representation theorem for the dual space to $C(Y, N)$, where $Y$ is compact Hausdorff and $N$ is a Banach space. This theorem is due to I. Singer [16] (see also [3]) and goes as follows: The space $C(Y, N)^{*}$ is isometrically isomorphic to the space of all countably additive regular measures of finite variation which are defined on the Borel subsets of $Y$ and take their values in $N^{*}$. The isometric isomorphism indicated makes each member of $C(Y, N)$ * correspond to an integral with respect to a member of the measure space. The latter space is normed by: $\|\mu\|=|\mu|(Y)$, where $|\mu|$ denotes the total variation of $\mu$. For the sake of clarity, we recall the definitions of some of the above terms. If $\mu$ is a finitely additive set function defined on the Borel subsets of $Y$ and taking values in $N^{*}$, then 
(1) If $B \subseteq Y$ is a Borel set, the total variation, $|\mu|(B)$, of $\mu$ at $B$ is

$$
|\mu|(B)=\sup \left\{\sum_{1}^{n}\left\|\mu\left(B_{i}\right)\right\|: B_{1}, \ldots, B_{n}\right. \text { are pairwise }
$$

disjoint Borel sets and $\left.B=\bigcup_{1}^{n} B_{i}\right\}$.

$\mu$ is said to be of finite variation if $|\mu|(Y)<\infty$.

(2) $\mu$ is said to be countably additive if, whenever $B_{1}, B_{2}, \ldots$ are pairwise disjoint Borel sets, we have $\mu\left(\bigcup B_{i}\right)=\sum \mu\left(B_{i}\right)$ where the right-hand side converges in norm.

(3) $\mu$ is regular if $B \rightarrow\langle\mu(B), x\rangle$ is a regular scalar-valued measure, for each $x \in N$.

(4) For a simple function $h=\sum_{1}^{n} x_{i} \chi_{B_{i}}$ from $Y$ into $N$, the integral is

$$
\int h d \mu=\sum_{1}^{n}\left\langle\mu\left(B_{i}\right), x_{i}\right\rangle \text {. }
$$

If $f: Y \rightarrow N$ is a uniform limit of a sequence $\left\{h_{n}\right\}$ of simple functions, then

$$
\int f d \mu=\lim \int h_{n} d \mu
$$

and this limit is independent of the choice of the sequence $\left\{h_{n}\right\}$.

Our next result is analogous to Theorem 2.1 and yields corollaries about compact operators in the norm topology in much the same way that theorem did for bounded operators in the weak-operator topology.

THEOREM 4.1. Let $Y$ be a compact Hausdorff space and let $M$ be a Banach space. If $A$ is a bounded subset of $M$ such that $(Y, A)$ has property (D), then $\operatorname{co}^{-} C(Y, A)$ $=C\left(Y, \mathrm{co}^{-} A\right)$, both closures being taken in the appropriate norm topologies.

Note. The same result was obtained by G. L. Seever [15] under the stronger hypothesis that $A$ is bounded, circled, and arcwise connected. Our proof was suggested by his.

Proof. Clearly, co- $C(Y, A) \subseteq C\left(Y\right.$, co $\left.^{-} A\right)$, since the latter is a closed convex set containing $C(Y, A)$. It suffices then to show that if $\mu$ is in $C(Y, M)^{*}$, with $\|\mu\|=1$, if $g \in C\left(Y, \operatorname{co}^{-} A\right)$, and if $\varepsilon>0$, then there exists $f \in C(Y, A)$ such that

$$
\operatorname{Re} \mu(g)<\operatorname{Re} \mu(f)+\varepsilon .
$$

Let $\delta \geqq 1$ be an upper bound for the norms of the elements of $A$; the same bound works also for $\mathrm{co}^{-} A$. Since $g(Y)$ is compact, we can choose $x_{1}, x_{2}, \ldots, x_{n} \in g(Y)$ and pairwise disjoint Borel sets $B_{1}, \ldots, B_{n}$ in $Y$ such that $Y=\bigcup B_{i}$ and

$$
\left\|g-\sum_{1}^{n} x_{i} \chi_{B_{i}}\right\|<\varepsilon / 4 \text {. }
$$


Since $g(Y) \subseteq \operatorname{co}^{-} A$, we can (by altering the $x_{i}$ 's slightly) assume $x_{1}, \ldots, x_{n} \in \operatorname{co} A$. Since $\|\mu\|=1$, we have

$$
\operatorname{Re} \mu(g)<\operatorname{Re} \mu\left(\sum x_{i} \chi_{B_{i}}\right)+\varepsilon / 4=\sum \operatorname{Re}\left\langle\mu\left(B_{i}\right), x_{i}\right\rangle+\varepsilon / 4 .
$$

Consider now the element $x_{1}$ of co $A$. It has the form $x_{1}=\sum \lambda_{k} y_{k}$, where $\lambda_{k}>0$, $\sum \lambda_{k}=1$, and each $y_{k}$ is in $A$. Since

we must have

$$
\operatorname{Re}\left\langle\mu\left(B_{1}\right), x_{1}\right\rangle=\sum \lambda_{k} \operatorname{Re}\left\langle\mu\left(B_{1}\right), y_{k}\right\rangle,
$$

$$
\operatorname{Re}\left\langle\mu\left(B_{1}\right), y_{k}\right\rangle \geqq \operatorname{Re}\left\langle\mu\left(B_{1}\right), x_{1}\right\rangle
$$

for some $y_{k}$; denote this element $y_{k} \in A$ by $z_{1}$. Similarly, choose elements $z_{2}, \ldots, z_{n}$ of $A$ corresponding (respectively) to $x_{2}, \ldots, x_{n}$. If we then let $h=\sum z_{i} \chi_{B_{i}}$, it is clear that $h(Y) \subseteq A$ and $\operatorname{Re} \mu(g)<\operatorname{Re} \mu(h)+\varepsilon / 4$. By regularity of $\mu$, we can choose a compact subset $K_{i}$ of $B_{i}$ such that

$$
|\mu|\left(B_{i} \backslash K_{i}\right)<\varepsilon / 4 n \delta, \quad i=1,2, \ldots, n .
$$

Let $K_{0}=\bigcup K_{i}$ and $U_{0}=Y \backslash K_{0}$. Then

$$
|\mu|\left(U_{0}\right)=|\mu|\left(Y \mid \cup K_{i}\right)=|\mu|\left(\bigcup\left(B_{i} \mid K_{i}\right)\right)=\sum|\mu|\left(B_{i} \mid K_{i}\right)<\varepsilon / 4 \delta .
$$

Property (D) implies that there exists $f$ in $C(Y, A)$ such that

$$
\left\|f(y)-z_{i}\right\|<\varepsilon / 4 \delta, \quad y \in K_{i}, \quad i=1,2, \ldots, n .
$$

Thus $\|f(y)-h(y)\|<\varepsilon / 4 \delta$, for all $y \in K_{0}$, and $\|f\| \leqq \delta$. Since $\|h\| \leqq \delta$, also, we have $\|h-f\| \leqq 2 \delta$ and

$$
\begin{aligned}
\operatorname{Re} \mu(h)-\operatorname{Re} \mu(f) \leqq\left|\int_{Y}(h-f) d \mu\right| & \leqq\left|\int_{K_{0}}(h-f) d \mu\right|+\left|\int_{U_{0}}(h-f) d \mu\right| \\
& \leqq \varepsilon / 4 \delta+(\varepsilon / 4 \delta) 2 \delta<3 \varepsilon / 4
\end{aligned}
$$

so that $\operatorname{Re} \mu(g)<\operatorname{Re} \mu(f)+\varepsilon$. This completes the proof.

As a corollary, we can immediately obtain a result about the unit ball of a space of type $C(Y, M)$.

Corollary 4.2. Let $Y$ be a compact Hausdorff space and let $M$ be a Banach space. If $S(M)$ is the norm-closed convex hull of its extreme points and if ( $Y$, ext $S(M)$ ) (norm topology on ext $S(M)$ ) has property (D), then the unit ball of $C(Y, M)$ is the norm-closed convex hull of its extreme points.

Proof. Simply observe that

$$
\begin{aligned}
\mathrm{co}^{-} \operatorname{ext} S(C(Y, M)) & \supseteq \mathrm{co}^{-} C(Y, \text { ext } S(M))=C\left(Y, \mathrm{co}^{-} \operatorname{ext} S(M)\right) \\
& =C(Y, S(M))=S(C(Y, M)) .
\end{aligned}
$$

The same conclusion was obtained by Bade [1] in case the scalars are real, $M$ is the scalar field, and $Y$ is totally disconnected. Later Phelps [13] proved the result 
for complex scalars, $M$ the scalar field, and $Y$ arbitrary. N. T. Peck [9] then proved it for $n$-dimensional euclidean space $(n>1)$, and $Y$ arbitrary. Next, Lindenstrauss (see [9]) improved Peck's result by allowing $M$ to be any finite-dimensional space such that the set of extreme points of the unit ball of $M$ is arcwise connected. Finally, the result of Seever mentioned following the statement of Theorem 4.1 yields the same result for arbitrary $Y$, assuming that the extreme points of $M$ are arcwise connected and norm generate the unit ball of $M$. Our result (with the help of Proposition 1.3) clearly implies all of the above. (However, in case $M$ is infinite dimensional and strictly convex (so that ext $S(M)$ is arcwise connected) and $Y$ is arbitrary, N. T. Peck [9] proved the much stronger conclusion that the unit ball in $C(Y, M)$ is actually the convex hull of its extreme points. In this connection, we mention also the following result due to Cantwell [17] and, in part, to Peck [9]: If $M$ is euclidean $n$-space, with $n>1$, and $Z$ is any normal Hausdorff space, then the unit ball of $C(Z, M)$ is the convex hull of its extreme points if and only if $Z$ has topological dimension $\leqq n-1$.)

When applied to compact operators, Theorem 4.1 yields the following:

Lemma 4.3. Let $M$ be a Banach space and let $Y$ be a compact Hausdorff space. If $\left(Y\right.$, ext $S\left(M^{*}\right)$ ) has property (D) (norm topology on ext $S\left(M^{*}\right)$ ) and if $S\left(M^{*}\right)$ is the norm-closed convex hull of its extreme points, then $S(K(M, C(Y)))$ is the norm-closed convex hull of its extreme points.

Proof. Under the identification between $C\left(Y, M^{*}\right)$ and $K(C(Y), M)$, ext $S(K)$ $\supseteq C\left(Y\right.$, ext $\left.S\left(M^{*}\right)\right)$ and $S(K)=C\left(Y, S\left(M^{*}\right)\right)$. Thus, by Theorem 4.1,

$$
\begin{gathered}
\operatorname{co}^{-} \operatorname{ext} S(K) \supseteq \operatorname{co}^{-} C\left(Y, \operatorname{ext} S\left(M^{*}\right)\right)=C\left(Y, \mathrm{co}^{-} \operatorname{ext} S\left(M^{*}\right)\right) \\
=C\left(Y, S\left(M^{*}\right)\right)=S(K) \supseteq \mathrm{co}^{-} \operatorname{ext} S(K),
\end{gathered}
$$

which proves the lemma.

We have immediately:

THEOREM 4.4. Let $M$ be a Banach space and let $Y$ be a compact Hausdorff space. Then $S(K(M, C(Y)))$ is the norm-closed convex hull of its extreme points if $S\left(M^{*}\right)$ is the norm-closed convex hull of its extreme points and if either:

(1) ext $S\left(M^{*}\right)$ is norm almost arcwise connected (which holds if $M^{*}$ is strictly convex): or

(2) $Y$ is totally disconnected.

In case $M$ is a space of type $C(X)$, our results are quite complete. First, we can completely characterize the extreme compact operators.

THEOREM 4.5. Let $X$ and $Y$ be compact Hausdorff spaces. Then every extreme member of $S(K(C(X), C(Y)))$ is nice.

Proof. Let $T \in \operatorname{ext} S(K)$. For each $y$ in $Y$, let $\mu_{y}=T^{*} y \in C(X)^{*}$. The function 
$y \rightarrow \mu_{y}$ is norm-continuous and is, indeed, an extreme point of the unit ball of $C\left(Y, C(X)^{*}\right)$. For each $y$ in $Y$, we can write $\mu_{y}=h_{y}\left|\mu_{y}\right|$, where $\left|\mu_{y}\right|$ is the total variation measure for $\mu_{y}$, and $h_{y}$ is a Borel function with $\left|h_{y}\right|=1$ a.e. $-\left|\mu_{y}\right|$. Without loss of generality, we can assume $\left|h_{y}\right|=1$ on all of $X$. To prove that $T$ is a nice compact operator, it suffices to show that $\left|\mu_{y}\right|$ is a point-evaluation measure for each $y \in Y$. We first show that $\left|\mu_{y}\right|$ has norm 1. Certainly, $\left\|\left|\mu_{y}\right|\right\|=\left\|\mu_{y}\right\| \leqq\|T\|=1$. Now

$$
\left\|\mu_{y} \pm\left(1-\left\|\mu_{y}\right\|\right) \mu_{y}\right\| \leqq\left\|\mu_{y}\right\|+\left(1-\left\|\mu_{y}\right\|\right)\left\|\mu_{y}\right\| \leqq\left\|\mu_{y}\right\|+\left(1-\left\|\mu_{y}\right\|\right)=1 .
$$

Since $y \rightarrow \mu_{y}$ is extreme and since $y \rightarrow\left(1-\left\|\mu_{y}\right\|\right) \mu_{y}$ is norm-continuous, $\left(1-\left\|\mu_{y}\right\|\right) \mu_{y}$ $=0$ for all $y \in Y$. We conclude that, for each $y$, either $\mu_{y}=0$ or $\left\|\mu_{y}\right\|=1$. We must show that the first possibility does not occur. Let $Y_{0}=\left\{y \in Y: \mu_{y}=0\right\}$. Then $Y_{0}$ is an open and closed set in $Y$. Let $x_{0} \in X$ and define $S f=f\left(x_{0}\right)_{\chi_{Y_{0}}}$, for all $f \in C(X)$. Then $S$ is a compact operator from $C(X)$ to $C(Y)$ and, clearly, $\|T \pm S\| \leqq 1$. Since $T$ is extreme, we conclude that $S=0$. Therefore, $Y_{0}=\varnothing$ and $\left|\mu_{y}\right|$ has norm 1 for all $y \in Y$. Now, the map $\lambda \rightarrow|\lambda|$ in $C(X)^{*}$ is norm-continuous and, therefore, the map $y \rightarrow\left|\mu_{y}\right|$ is norm-continuous. Suppose, for the moment, that we have shown that $y \rightarrow\left|\mu_{y}\right|$ is extreme in the (convex) set of all norm-continuous maps from $Y$ into the set of probability measures on $X$. The corresponding set of operators are those positive compact operators from $C(X)$ to $C(Y)$ which carry 1 to 1 . From the proof of Theorem 1.1 of [12], it follows that the extreme operator corresponding to $y \rightarrow\left|\mu_{y}\right|$ is multiplicative, so that $\left|\mu_{y}\right|$ is multiplicative and hence a point-evaluation measure, for each $y \in Y$. Thus, our proof will be complete as soon as we verify our assumption concerning $y \rightarrow\left|\mu_{y}\right|$. Suppose, on the contrary, that there exists a norm-continuous map $y \rightarrow \nu_{y}$ such that $\left\|\left|\mu_{y}\right| \pm \nu_{y}\right\|=1$ and $\left|\mu_{y}\right| \pm \nu_{y} \geqq 0$ for each $y \in Y$, and $\nu_{y_{0}} \neq 0$ for some $y_{0} \in Y$. For each $y \in Y$, let $\lambda_{y}=h_{y} \nu_{y}$. Since, clearly, $\lambda_{y_{0}} \neq 0$, we will have obtained a contradiction to $y \rightarrow \mu_{y}$ being extreme if we show that $y \rightarrow \lambda_{y}$ is continuous and that $\left\|\mu_{y} \pm \lambda_{y}\right\| \leqq 1$. Now, since $\left|h_{y}\right|=1$ for each $y$, we have

$$
1=\left\|\left|\mu_{y}\right| \pm \nu_{y}\right\|=\left\|h_{y}\left(\left|\mu_{y}\right| \pm \nu_{y}\right)\right\|=\left\|\mu_{y} \pm \lambda_{y}\right\| .
$$

To see that $y \rightarrow \lambda_{y}$ is norm-continuous, we first observe that for any bounded Borel function $h$ on $X$, we have

$$
|h|\left(\left|\mu_{y}\right| \pm \nu_{y}\right) \geqq 0, \quad \forall y \in Y,
$$

and hence $\left\|h \nu_{y}\right\|=\left\||h| \nu_{y}\right\| \leqq\left\||h|\left|\mu_{y}\right|\right\|=\left\|h\left|\mu_{y}\right|\right\|$. Consequently, if $y, z$ are in $Y$ then

$$
\begin{aligned}
\left\|\lambda_{y}-\lambda_{z}\right\| & =\left\|h_{y} \nu_{y}-h_{z} \nu_{z}\right\| \leqq\left\|\left(h_{y}-h_{z}\right) \nu_{y}\right\|+\left\|h_{z}\left(\nu_{y}-\nu_{z}\right)\right\| \\
& \leqq\left\|\left(h_{y}-h_{z}\right)\left|\mu_{y}\right|\right\|+\left\|\nu_{y}-\nu_{z}\right\| \\
& \leqq\left\|h_{y}\left|\mu_{y}\right|-h_{z}\left|\mu_{z}\right|\right\|+\left\|h_{z}\left(\left|\mu_{y}\right|-\left|\mu_{z}\right|\right)\right\|+\left\|\nu_{y}-\nu_{z}\right\| \\
& \leqq\left\|\mu_{y}-\mu_{z}\right\|+\left\|\left|\mu_{y}\right|-\left|\mu_{z}\right|\right\|+\left\|\nu_{y}-\nu_{z}\right\| .
\end{aligned}
$$


Since the maps $y \rightarrow \mu_{y}, y \rightarrow\left|\mu_{y}\right|$, and $y \rightarrow \nu_{y}$ are all norm-continuous, so is $y \rightarrow \lambda_{y}$, and the proof is complete.

THEOREM 4.6. Let $X$ and $Y$ be compact Hausdorff spaces. (In case the scalars are complex, assume that if $Y$ has at least 2 points, then so does $X$.) Then $S(K(C(X), C(Y)))$ is the norm-closed convex hull of its extreme points if and only if $Y$ is totally disconnected and $X$ contains no nonvoid perfect subsets.

Proof. Assume $Y$ is totally disconnected and $X$ contains no nonvoid perfect subsets. By a theorem of Pełczyński and Semadeni [11], $S\left(C(X)^{*}\right)$ is the normclosed convex hull of its extreme points. Hence, $S(K)$ is the norm-closed convex hull of its extreme points, by Theorem 4.4 .

Now suppose $S(K)=$ co $^{-}$ext $S(K)$. We first show that $Y$ is totally disconnected. Suppose, on the contrary, that $D$ is a connected subset of $Y$ containing more than one point. Let $T$ be an extreme compact operator. Then, by Theorem 4.5, $T$ is nice and hence $y \rightarrow \mu_{y}$ (notation as in previous proof) carries $Y$ (norm-continuously) into ext $S\left(C(X)^{*}\right)$. Now, in the norm topology, ext $S\left(C(X)^{*}\right)=X_{d} \times E$, where $X_{d}$ is $X$ with the discrete topology and $E$ is the set of scalars with absolute value 1 . Since $D$ is connected, $\left|\mu_{y}\right|=\left|\mu_{z}\right|$ whenever $y, z \in D$, i.e. $\mu_{y}$ and $\mu_{z}$ are (perhaps different) multiples of the same point-evaluation functional. Now let $x_{1}, x_{2}$ be distinct points of $X$ and choose $f_{1}, f_{2}$ in $C(X)$ such that $f_{i}\left(x_{i}\right)=1=\left\|f_{i}\right\|$, $i=1,2$, and such that $f_{1}$ and $f_{2}$ have disjoint supports. Then let $y_{1}, y_{2}$ be distinct points of $D$ and define $\varphi(S)$, for $S \in K$, by:

$$
\varphi(S)=\left(S f_{1}\right)\left(y_{1}\right)+\left(S f_{2}\right)\left(y_{2}\right) .
$$

Then $\varphi$ is a continuous linear functional on the space $K$. We have $\operatorname{Re} \varphi(T) \leqq 1$, whenever $T \in \operatorname{ext} S(K)$ and, hence, whenever $T \in \mathrm{co}^{-} \operatorname{ext} S(K)$. Therefore, to obtain a contradiction, it suffices to produce $S \in S(K)$ such that $\operatorname{Re} \varphi(S)>1$. To do this, let $h_{1}, h_{2}$ be in $C(Y)$ such that $0 \leqq h_{i} \leqq 1, h_{i}\left(y_{i}\right)=1, i=1,2$, and such that $h_{1}+h_{2} \leqq 1$. Then define

$$
(S f)(y)=h_{1}(y) f\left(x_{1}\right)+h_{2}(y) f\left(x_{2}\right),
$$

for $f$ in $C(X), y$ in $Y$. Clearly, $S$ is a compact operator with $\|S\| \leqq 1$. Also

$$
\varphi(S)=h_{1}\left(y_{1}\right) f_{1}\left(x_{1}\right)+h_{2}\left(y_{1}\right) f_{1}\left(x_{2}\right)+h_{1}\left(y_{2}\right) f_{2}\left(x_{1}\right)+h_{2}\left(y_{2}\right) f_{2}\left(x_{2}\right)=2 .
$$

This completes the proof that $Y$ is totally disconnected, provided, of course, that it was possible to choose 2 distinct points in $X$. If $X$ consists of a single point and the scalars are complex, then, by hypothesis, $Y$ is also a singleton and therefore totally disconnected. If the scalars are real, our assertion reduces to the known result [1] that $Y$ is totally disconnected if the unit ball of real $C(Y)$ is the normclosed convex hull of its extreme points.

We now show that $X$ contains no nonvoid perfect subsets. Pełczyński and 
Semadeni [11] also proved the converse of their result quoted earlier and, therefore, we need only show that $S\left(C(X)^{*}\right)=$ co- $^{-}$ext $S\left(C(X)^{*}\right)$. We have

$$
\begin{aligned}
C\left(Y, S\left(C(X)^{*}\right)\right) & \equiv S(K)=\mathrm{co}^{-} \operatorname{ext} S(K) \\
& \equiv \mathrm{co}^{-} C\left(Y, \operatorname{ext} S\left(C(X)^{*}\right)\right) \\
& \subseteq C\left(Y, \mathrm{co}^{-} \operatorname{ext} S\left(C(X)^{*}\right) .\right.
\end{aligned}
$$

Therefore, $S\left(C(X)^{*}\right) \subseteq$ co- $^{-}$ext $S\left(C(X)^{*}\right)$, and the proof is complete.

In analogy with the noncompact case (Theorem 3.1), one would hope to be able to prove that, if $S(K(M, C(Y)))$ is the norm-closed convex hull of the nice compact operators, then $C\left(Y\right.$, ext $S\left(M^{*}\right)$ ) (norm topology on ext $S\left(M^{*}\right)$ ) is pointwise dense in (ext $\left.S\left(M^{*}\right)\right)^{Y}$. Unfortunately, we are not able to do so. We can, however, prove the following weaker assertion:

Proposition 4.7. Let $Y$ be a compact Hausdorff space and let $M$ be a Banach space. Suppose $S(K(M, C(Y)))$ is the norm-closed convex hull of the nice compact operators. If $U_{1}, U_{2}, \ldots, U_{n}$ are nonvoid weak* open subsets of ext $S\left(M^{*}\right)$ and $y_{1}, y_{2}, \ldots, y_{n}$ are distinct points of $Y$, then there exists a norm-continuous map $f: Y \rightarrow \operatorname{ext} S\left(M^{*}\right)$ such that $f\left(y_{i}\right) \in U_{i}$, for all $i$.

Proof. The argument is much like the one for Theorem 3.1. Choose $\delta>0$ and $x_{1}, x_{2}, \ldots, x_{n} \in M$ with $\left\|x_{i}\right\|=1, i=1,2, \ldots, n$, such that

$$
\left\{x^{*} \in \operatorname{ext} S\left(M^{*}\right): \operatorname{Re}\left\langle x^{*}, x_{i}\right\rangle>1-\delta\right\} \subseteq U_{i}, \quad i=1,2, \ldots, n .
$$

Define $\mu \in C\left(Y, M^{*}\right)^{*}$ by:

$$
\mu(g)=\sum_{1}^{n}\left\langle g\left(y_{i}\right), x_{i}\right\rangle
$$

for all $g \in C\left(Y, M^{*}\right)$. Then $\|\mu\|=n$ and we can find $f$ in $C\left(Y\right.$, ext $\left.S\left(M^{*}\right)\right)$ such that $\operatorname{Re} \mu(f)>\|\mu\|-\delta=n-\delta$. But the left-hand side of the above inequality is

$$
\operatorname{Re} \sum_{1}^{n}\left\langle f\left(y_{i}\right), x_{i}\right\rangle \leqq n-1+\operatorname{Re}\left\langle f\left(y_{j}\right), x_{j}\right\rangle
$$

for all $j=1,2, \ldots, n$. Hence $\operatorname{Re}\left\langle f\left(y_{j}\right), x_{j}\right\rangle>1-\delta$. Therefore $f\left(y_{j}\right) \in U_{j}, j=1,2, \ldots$, $n$, and the proof is complete.

The following is immediate:

Corollary 4.8. Let $Y, M$ be as above and suppose $S(K(M, C(Y)))$ is the normclosed convex hull of the nice compact operators. Then

(1) if ext $S\left(M^{*}\right)$ is not weak* connected, then $Y$ is totally disconnected;

(2) if $Y$ is infinite and almost arcwise connected, then, given $U_{1}, U_{2}, \ldots, U_{n}$, nonvoid, weak* open subsets of ext $S\left(M^{*}\right)$, there exists a norm-continuous

$$
f:[0,1] \rightarrow \operatorname{ext} S\left(M^{*}\right)
$$

such that $f(i / n) \in U_{i}, i=1,2, \ldots, n$. 
5. Extension operators. Let $X$ be a closed subset of a compact Hausdorff space $Y$. The set $\mathscr{E}=\mathscr{E}(X, Y)$ of positive extension operators of norm 1 from $C(X)$ into $C(Y)$ (i.e. those $T$ in $K_{0}(X, Y)$ such that $T f$ is an extension of $f$ for each $f$ in $C(X)$ ) is (if nonempty) convex and weak-operator closed. The set $\mathscr{E}$ is not always nonvoid, but it is when $X$ is metrizable (see $\S 6$ of [10] and the references cited there for more information). It is easily seen that $\mathscr{E}$ is an extremal subset of $K_{0}$. Hence ext $\mathscr{E}$ $=\mathscr{E} \cap$ ext $K_{0}=\left\{T\right.$ in $\left.\mathscr{E}: T^{*}[Y] \subseteq X \cup\{0\}\right\}$, where we have used the characterization of ext $K_{0}$ mentioned before the statement of Corollary 2.6. It is convenient to rephrase this characterization of ext $\mathscr{E}$ as follows:

Proposition 5.1. Let $X$ and $Y$ be as above. For $T$ in $\mathscr{E}(X, Y)$, the following are equivalent:

(1) $T$ is in ext $\mathscr{E}$;

(2) there is an open compact subset $A$ of $Y \backslash X$ and a retraction $\rho$ of $Y \backslash A$ onto $X$ such that, for all $f$ in $C(X)$,

$$
\begin{aligned}
(T f)(y) & =f \circ \rho(y), & & y \text { in } Y \backslash A, \\
& =0, & & y \text { in } A .
\end{aligned}
$$

Thus ext $\mathscr{E}$ is nonvoid if and only if $X$ is a retract of $Y$.

The following useful result is a special case of Theorem $1^{\prime}$ of $[8$, p. 266]. For completeness, we include a short proof suggested by E. A. Michael.

Lemma 5.2. Let $W$ be a closed subset of a compact metric space $Z$. If $Z \backslash W$ is totally disconnected, then $W$ is a retract of $Z$.

Proof. For each $z$ in $Z \backslash W$, let $U_{z}$ denote the open ball about $z$ of radius $\frac{1}{2} d(z, W)$. Choose a pairwise disjoint open and closed refinement $\left\{K_{\alpha}\right\}$ of the open cover $\left\{U_{z}: z\right.$ in $\left.Z \backslash W\right\}$ of $Z \backslash W$, and for each $\alpha$, let $w_{\alpha}$ be a nearest point in $W$ to $K_{\alpha}$. Define $\rho(z)=z$ for $z$ in $W$ and $\rho(z)=w_{\alpha}$ for $z$ in $K_{\alpha}$. The map $\rho$ is easily seen to be continuous.

Our result, the proof of which is omitted, is the following:

THEOREM 5.3. Let $X$ be a closed subset of a compact metric space $Y$. Then $\mathscr{E}(X, Y)$ is the weak operator-closed convex hull of its extreme points if and only if $Y \backslash X$ is totally disconnected.

The proof of the "if" half is similar to the proof of Theorem 2.1, while the proof of the "only if" half is similar to the second part of the proof of Corollary 2.7.

\section{REFERENCES}

1. W. Bade, Functional analysis seminar notes (unpublished), Univ. of California, Berkeley, 1957.

2. R. M. Blumenthal, J. Lindenstrauss and R. R. Phelps, Extreme operators into $C(K)$, Pacific J. Math. 15 (1965), 747-756. MR 35 \#758.

3. N. Dinculeanu, Vector measures, Internat. Series of Monographs in Pure and Appl. 
Math., vol. 95, Pergamon Press, New York and VEB Deutscher Verlag, Berlin, 1967. MR 34 \#6011b.

4. N. Dunford and J. T. Schwartz, Linear operators. Part 1: General theory, Pure and Appl. Math., vol. 7, Interscience, New York, 1958. MR 22 \#8302.

5. A. Ionescu Tulcea and C. Ionescu Tulcea, $A$ note on extreme points (unpublished).

6. —_, On the lifting property. I, J. Math. Anal. Appl. 3 (1961), 537-546. MR 27 \#257.

7. J. L. Kelley, General topology, Van Nostrand, Princeton, N. J., 1955. MR 16, 1136.

8. C. Kuratowski, Topologie. Vol. II, Warsaw, 3ième. ed., Monogr. Mat., Tom 21, PWN, Warsaw, 1961. MR 24 \#A2958.

9. N. T. Peck, Extreme points and dimension theory, Pacific J. Math. 25 (1968), 341-351.

10. A. Pełczyński, Linear extensions, linear averagings, and their applications to linear topological classification of spaces of continuous functions, Dissertationes Math. Rozprowy Mat. 58 (1968). MR 37 \#3335.

11. A. Pełczyński and Z. Semadeni, Spaces of continuous functions. III: Spaces $C(\Omega)$ for $\Omega$ without perfect subsets, Studia Math. 18 (1959), 211-222. MR 21 \#6528.

12. R. R. Phelps, Extreme positive operators and homomorphisms, Trans. Amer. Math. Soc. 108 (1963), 265-274. MR 27 \#6153.

13. — Extreme points in function algebras, Duke Math. J. 32 (1965), 267-277. MR 31 \#3890.

14. —, Lectures on Choquet's theorem, Van Nostrand, Princeton, N. J., 1966. MR 33 \#1690.

15. G. L. Seever, Generalization of a theorem of Lindenstrauss (mimeographed notes).

16. I. Singer, Linear functionals on the space of continuous mappings of a compact Hausdorff space into a Banach space, Rev. Math. Pures Appl. 2 (1957), 301-315. (Russian) MR 20 \#3445.

17. J. Cantwell, $A$ topological approach to extreme points in function spaces, Proc. Amer. Math. Soc. 19 (1968), 821-825. MR 37 \#4583.

18. M. A. Rieffel, A characterization of commutative group algebras and measure algebras, Bull. Amer. Math. Soc. 69 (1963), 812-814. MR 27 \#4092.

19. J. L. Taylor, The structure of convolution measure algebras, Trans. Amer. Math. Soc. 119 (1965), 150-166. MR 32 \#2932.

The Pennsylvania State University

University Park, Pennsylvania 16802

UNIVERSITY OF WASHINGTON,

SeATtLe, Washington 98105 